\title{
Three-step anti-aMPV IgA expression profile evaluation in turkeys of different immunological status after TRT vaccination
}

\author{
M. Śmiałek, B. Tykałowski, D. Pestka, J. Welenc, T. Stenzel, A. Koncicki \\ Department of Poultry Diseases, Faculty of Veterinary Medicine, University of Warmia and Mazury, \\ Oczapowskiego 13, 10-719 Olsztyn, Poland
}

\begin{abstract}
Maternally derived antibodies (MDA) don not protect turkeys against rhinotracheitis (TRT) but high MDA influences upper respiratory tract (URT) immunity stimulation after avian Metapneumovirus (aMPV) vaccination. Humoral immunity can not be considered as an indicator of protection against TRT, but specific antibodies inhibit aMPV replication and alleviate the course of TRT. Scarce reports indicate the role of IgA in protection against TRT. The aim of our study was to investigate the impact of MDA on stimulation, antigen specificity acquisition of B lymphocytes, and the production of specific IgA after TRT vaccination of turkeys.

The results of our study indicate that MDA on the day of TRT vaccination causes disturbances at different levels of specific humoral immunity expression including antigen specificity acquisition of $\mathrm{B} \mathrm{IgA}^{+}$lymphocytes as well as production and secretion of IgA. Vaccine immunity against aMPV associated with sIgA is well expressed in birds not possessing MDA on the day of TRT vaccination, whereas it is inhibited in $\mathrm{MDA}^{+}$birds.

These results corroborate our previous findings and indicate that MDA could be responsible for TRT vaccination failure. These findings could explain the observed frequency of TRT field outbreaks despite aMPV vaccination of turkey flocks.
\end{abstract}

Key words: aMPV, turkeys, vaccination, IgA; ELISPOT, maternally derived antibodies

\section{Introduction}

Avian Metapneumovirus (aMPV), RNA virus being a member of the Paramyxoviridae, genus Metapneumovirus (Buys and Preez 1980, Pedersen et al. 2000), is a causative agent of turkey rhinotracheitis (TRT). aMPV has a worldwide distribution and only Australia and Canada are still considered free from
TRT. Up to date aMPV have been classified into 4 subtypes (A - D) (Collins et al. 1993, Cook et al. 1993).

Turkeys respond with strong stimulation of humoral immunity to aMPV infection; however, there is a lack of correlation between specific antibodies and protection against TRT (Jones et al. 1988, Cook et al. 1989, Cha et al. 2007). On the other hand, the clinical

Correspondence to: M. Śmiałek, e-mail: marcin.smialek@uwm.edu.pl 
Table 1. Mean $x$-fold change of $\operatorname{IgA}^{+}$B ACC at days post vaccination ${ }^{\mathrm{a}}$ in $\mathrm{HG}$ and TC and MDA IgY levels on the day of aMPV vaccination.

\begin{tabular}{|c|c|c|c|c|c|c|}
\hline \multirow{2}{*}{ Experiment } & \multirow{2}{*}{ 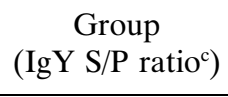 } & \multirow{2}{*}{ Organ } & \multicolumn{4}{|c|}{ Mean $x$-fold change ${ }^{b}$} \\
\hline & & & $0 \mathrm{DPV}$ & $3 \mathrm{DPV}$ & $7 \mathrm{DPV}$ & $14 \mathrm{DPV}$ \\
\hline \multirow[t]{2}{*}{$\mathrm{I}$} & $\mathrm{MDA}^{+} 0 / \mathrm{V}$ & HG & 1 & $1.81 *$ & $2.09 * *$ & $1.47 *$ \\
\hline & $(1.152)$ & $\mathrm{TC}$ & 1 & 0.81 & 0.86 & 1.20 \\
\hline \multirow[t]{2}{*}{ II } & $\mathrm{MDA}^{+} 14 / \mathrm{V}$ & HG & 1 & 1.42 & $2.09 * *$ & 1.03 \\
\hline & $(0.128)$ & $\mathrm{TC}$ & 1 & 1.27 & 1.32 & 1.29 \\
\hline \multirow[t]{2}{*}{ III } & MDA- $0 / \mathrm{V}$ & HG & 1 & 0.62 & 1.15 & $1.99 * *$ \\
\hline & $(0.0)$ & $\mathrm{TC}$ & 1 & 1.31 & 0.91 & $1.66 * *$ \\
\hline \multirow[t]{2}{*}{ IV } & MDA-14/V & $\mathrm{HG}$ & 1 & 0.88 & 0.96 & 0.89 \\
\hline & $(0.0)$ & $\mathrm{TC}$ & 1 & 1.07 & $1.63 *$ & $2.41 * *$ \\
\hline
\end{tabular}

${ }^{a}$ Birds were inoculated oculonasaly with $10^{4} \mathrm{TCID}_{50}$ of live attenuated aMPV/A vaccine

${ }^{\mathrm{b}} x$-fold change $=$ mean $\operatorname{IgA}^{+} \mathrm{B}$ ACC of vacc. group $/$ mean $\mathrm{IgA}^{+} \mathrm{B} \mathrm{ACC}$ of not vacc. group

*** Significant difference in mean $\operatorname{IgA}^{+}$B ACC of vaccinated birds in comparison to the not vaccinated group (T-test, $*$ as $\mathrm{p}<0.05$ and $* *$ as $\mathrm{p}<0.01$ )

${ }^{\mathrm{c}}$ MDA level on the day of TRT vaccination. Results are expressed as mean S/P ratio $(\mathrm{n}=15)$

course of TRT in birds subjected to B lymphocyte depletion was more severe in comparison with fully immunocompetent birds after TRT challenge (Jones et al. 1992). The above indicates that humoral immunity does not protect birds against the disease, but it alleviates the course of TRT.

Humoral immunity of the body mucous membranes is associated with $\operatorname{IgA}$ (also reffered to as secretory IgA - sIgA) (Snoeck et al. 2006, Smialek et al. 2011).

Little is known about the role of IgA in the protection against TRT. Cha et al. (2007) demonstrated an increase of infiltrating $\operatorname{Ig} \mathrm{A}^{+} \mathrm{B}$ cells in nasal turbinates 7 days after aMPV/C exposure, with an elevated anti-aMPV IgA level detected in the nasal secretions after aMPV/C re-exposure. These results indicate that $\operatorname{SIgA}$ is involved in protection against TRT and that these antibodies are responsible for suppression of aMPV replication in the upper respiratory tract (URT), and therefore they alleviate the course of TRT and enable faster recovery.

Considering the above, and our previous findings concerning the differences in humoral and cell-mediated immunity stimulation after TRT vaccination of turkeys with or without maternally derived antibodies (MDA) (Smialek et al. 2015), the aim of this study was to investigate the impact of MDA on stimulation and antigen memory acquisition of $\mathrm{B} \operatorname{IgA}^{+}$cells and the production of anti-aMPV IgA in the URT after TRT vaccination of turkeys.

\section{Materials and Methods}

The animal protocol and animal handling procedures were approved by the Local Ethics Commit- tee for Animal Experiments in Olsztyn, Poland (resolution No. 37/2011).

\section{Turkeys and vaccination}

568 commercial Hybrid Converter turkeys were used in the experiments. The birds were not vaccinated at the hatchery against TRT, which was confirmed with RT-PCR (Smialek et al. 2015) in URT swabs collected during experiments (data not shown). One group of turkeys (50\%) (provided by the Grelavi S.A. hatchery [a Hendrix Genetics Company], Poland) that possessed MDA $\left(\mathrm{MDA}^{+}\right)$originated from breeder turkeys vaccinated against TRT (with live aMPV/A vaccine [3 times] and inactivated vaccine [twice]). The other group (provided by the Gerczak Nord-Pol Hatchery, Poland) that did not possess MDA (MDA-) originated from a Canadian breeder flock. Turkeys for all experiments were provided by the hatcheries at the same time.

The turkeys were housed in isolated units maintained at a PCL 3 (physical containment level 3) facility. They were vaccinated occulonasaly with $10^{4}$ of tissue infectious dose $50 \%$ (TCID $\left._{50}\right) /$ bird of aMPV/A strain BUT1 \#8544 commercial attenuated vaccine reconstituted in milli-q water. Non-vaccinated birds received vaccine diluent. Water and feed were given to birds ad libitum. IgY MDA status was confirmed with ELISA (IDEXX, USA) (Table 1).

\section{Experimental design}

Experiments I and III. 160 zero-day old $\mathrm{MDA}^{+}$ (Experiment I) or MDA- (Experiment III) turkeys 
were used. Birds for these experiments were randomly divided into appropriate ( $\mathrm{MDA}^{+}$or $\left.\mathrm{MDA}-\right)$ vaccinated $(0 / \mathrm{V})$ and not vaccinated $(0 / \mathrm{NV})$ groups. Before aMPV/A vaccination (0 day of life - DOL $=0$ day post vaccination - DPV) serum and tracheal washing (TW), Harderian glands (HG) and tracheas (TC) were collected for IgA serological examination and flow cytometry analyses from 24 randomly selected birds of either $\mathrm{MDA}^{+} 0 / \mathrm{NV}$ or MDA-0/NV groups. The birds were raised to 14 DOL, and further samples were collected on 3, 7 and 14 DPV.

After blood sampling from 15 birds of each group, 20-24 birds of each group were euthanized, and HG (entire organ bilaterally) and TC (cut from the larynx to syrinx) were collected. HG and TC were pooled from 5-6 birds per sample (in different groups the number of birds per sample was equal in each sampling), which produced 4 samples of each organ for flow cytometry and ELIPOT analysis. Tracheal washings were collected from 10 birds in each group as described previously (Smialek et al. 2015). Following centrifugation, the supernatant and serum samples were stored at $-20^{\circ} \mathrm{C}$ for ELISA.

Experiments II and IV. The experimental design was identical to that of Experiments I and III. Experiments were carried out on $124 \mathrm{MDA}^{+}(\mathrm{Ex}-$ periment II) or MDA- (Experiment IV) turkeys raised until 14 DOL and divided into vaccinated $(14 / \mathrm{V})$ and non-vaccinated (14/NV) groups. On 14 DOL, samples were collected from 20 birds of either $\mathrm{MDA}^{+} 14 / \mathrm{NV}$ or $\mathrm{MDA}-14 / \mathrm{NV}$ groups for serological and flow cytometry analyses. Similarly to Experiment I and III, the results reported for samples that were collected before vaccination (14 DOL) represent $0 \mathrm{DPV}$, and further samples were collected on 3 (17 DOL), 7 (21 DOL) and 14 (28 DOL) DPV (16 - 20 birds in each group in each sampling).

\section{Isolation and determination of mononuclear cell counts}

Isolation of mononuclear cells from $\mathrm{HG}$ and TC, with the use of Percoll density gradient solution, were performed as described previously (Smialek et al. 2015).

The obtained HG and TC mononuclear cells were resuspended in $1 \mathrm{ml}$ of PBS. Vi-cell XR (Beckman Coulter, USA) cell counter and cell viability analyzer was used for each sample to calculate the absolute lymphocyte counts (ALC) per ml.

\section{Flow cytometry and dual platform analysis}

$2.5 \times 10^{5}$ (or $1.25 \times 10^{5}$, depending on the ALC) of viable mononuclear cells from $\mathrm{HG}$ or TC were stained with polyclonal Goat anti-Chicken IgA-FITC (AbD Serotec, UK), incubated for $30 \mathrm{~min}$ on ice and washed twice in PBS. Goat anti-chicken IgA cross-reacting with turkey IgA (Cha et al. 2007) were used and their specificity was evaluated with the use of turkey lymphocytes isolated from the Bursa of Fabricius and thymus (data not shown). Finally, the cells were analyzed using a FACSCanto II (BD, USA) flow cytometer.

Further steps of flow cytometry data acquisition and dual platform analysis allowing the calculation of $\mathrm{B} \operatorname{IgA}^{+}$absolute cell count (ACC) were performed as described previously (Smialek et al. 2015). Data were expressed as mean $\mathrm{x}$-fold change in $\mathrm{ACC}$ of $\operatorname{IgA} \mathrm{A}^{+}$ $\mathrm{B}$ lymphocytes in vaccinated groups relative to non-vaccinated groups.

\section{Cell sorting and ELISPOT assay}

ELISPOT was performed as described previously (Van Ginkel et al. 2008) with modifications. After membrane activation (70\% ethanol, $50 \mu \mathrm{l} / \mathrm{well}, 45 \mathrm{~s})$ and 4 PBS wash cycles, MultiScreen plates (Millipore, USA) were coated with PBS solutions of vaccine aMPV/A or polyclonal goat anti-Chicken IgA antibodies $\left(4^{\circ} \mathrm{C}, 24 \mathrm{~h}\right)$. The plates were then washed 3 times with PBS-Tween 20 solution (0.05\%; Sigma Aldrich, Germany) and blocked with RPMI medium containing $20 \%$ fetal bovine serum $\left(1 \mathrm{~h}, 37^{\circ} \mathrm{C}\right)$.

HG and TC mononuclear cells were sorted with BD FACSAria II (BD, USA). A lymphocyte gate was established based on cell morphology, which enabled sorting without the necessity of cell staining (risk of unselective stimulation). $1 \times 10^{4}$ lymphocytes from HG or $0.5 \times 10^{4}$ lymphocytes from TC were sorted directly to the wells of previously prepared plates. Lymphocytes from each sample were sorted in duplicate (3 DOL) or triplicate (older birds) to wells coated with aMPV/A and anti-IgA antibodies respectively. Before incubation $\left(24 \mathrm{~h}, 39.5^{\circ} \mathrm{C}, 5 \% \mathrm{CO}_{2}\right), 100$ $\mu \mathrm{l}$ of Iscove's modified Dulbecos medium (Sigma Aldrich, Germany) was added to each well. After incubation, the plates were washed 4 times with PBS-Tween 20 and once with PBS and incubated overnight $\left(4^{\circ} \mathrm{C}\right)$ with goat anti-Chicken IgA:alkaline phosphatase. The plates were then washed 3 times with PBS, and after loosening the bottom of the plate, the membranes were washed with PBS on the reverse. Enzymatic reaction was performed with an alkaline phosphatase substrate (BCIP/NBT; Vector Labora- 
tories, USA) for $15-25$ minutes. The enzyme reaction was stopped with water. Counting of anti-aMPV $\mathrm{B} \operatorname{IgA}^{+}$(wells coated with aMPV/A) spot forming units (SFU) and Total $\mathrm{B} \operatorname{IgA}{ }^{+}$SFU (wells with anti - Chicken IgA) was performed with the use of an Eli.Scan plate scanner and Eli.Analyse software (A-EL-VIS, Germany).

The contribution of anti-aMPV IgA secreting $B$ cells was calculated with the use of the following formula: (mean anti-aMPV B $\mathrm{IgA}^{+} \mathrm{SFU} /$ mean total $\left.\mathrm{B} \mathrm{IgA}^{+} \mathrm{SFU}\right)^{*} 100 \%$. Data were expressed as mean $\mathrm{x}$-fold change of contribution of anti-aMPV IgA secreting $B$ cells in vaccinated groups relative to non-vaccinated groups per each group in each DPV.

\section{In-house Enzyme-linked immunosorbent assay (ELISA)}

The level of specific anti-aMPV IgA in serum and TW was determined with in-house ELISA. After 3 freeze - thaw cycles and purification with the use of $0.22 \mu \mathrm{m}$ syringe filters (Millipore, USA), vaccine aMPV/A diluted in Coating Buffer $\mathrm{B}(\mathrm{pH}=9.4$; Invitrogen, USA) was used to coat the maxisorp ELISA plates (NUNC, Denmark). The plates were incubated $\left(24 \mathrm{~h}, 4^{\circ} \mathrm{C}\right)$ and then washed 4 times with Wash Buffer (Invitrogen, USA) and blocked $\left(1 \mathrm{~h}, 21^{\circ} \mathrm{C}\right)$ with Assay Buffer (Invitrogen, USA). $100 \mu \mathrm{l}$ of serum samples (diluted 1:250 in Assay Buffer) and TW (undiluted) were incubated $\left(1 \mathrm{~h}, 21^{\circ} \mathrm{C}, 200 \mathrm{RPM}\right)$ on plates. After incubation, the plates were washed 4 times with Washing buffer $350 \mu \mathrm{l} /$ well. Policlonal anti - Chicken IgA:HRP antibodies $\left(1 \mathrm{~h}, 21^{\circ} \mathrm{C}, 200 \mathrm{RPM} ; \mathrm{AbD}\right.$ Serotec, UK) and HRP substrate $\left(15 \mathrm{~m}, 21^{\circ} \mathrm{C}\right.$; Invitrogen, USA) were used in further steps of the in-house ELISA method. Stop Solution (Invitrogen, USA) was used to terminate the enzymatic reaction. In-house ELISA was carried out with a BioTek ELx405 automatic plate washer (BioTek, USA), Eppendorf epMotion $5075 \mathrm{LH}$ pipetting station (Eppendorf, Germany), and a BioTek ELx800 plate reader. Data were expressed as mean OD +/- SD in each group in each DPV. Optimal dilutions of vaccine aMPV/A, conjugate, serum and TW in ELISA and ELISPOT assays were determined with their previous titration with the use of immunocompetent cells, serum and TW samples from non-vaccinated (negative control samples) and hyper-immunized birds (positive control samples). ELISPOT and ELISA were validated and optimized with negative control wells (not coated with either aMPV/A or goat anti-chicken IgA - data not shown).

\section{Statistical analysis}

The results were processed by Student's t-test for independent samples in Graphpad Prism 6 software (San Diego, USA). Differences were considered statistically significant at $\mathrm{p} \leq 0.05$ and highly significant at $\mathrm{p} \leq 0.01$

\section{Results}

\section{Flow cytometry analysis of B cells}

In Experiment I, a statistically highly significant or significant increase in $\operatorname{IgA}^{+} \mathrm{B}$ ACC was noted in the $\mathrm{MDA}^{+} 0 / \mathrm{V}$ group on days 3,7 and $14 \mathrm{PV}$ in $\mathrm{HG}$, in comparison with virus-free birds (Table 1).

In Experiment II a highly significant increase in $\mathrm{IgA}^{+} \mathrm{B}$ ACC was observed in the $\mathrm{MDA}^{+} 14 / \mathrm{V}$ group on day $7 \mathrm{PV}$ in $\mathrm{HG}$, in comparison with control birds (Table 1)

No statistical differences in the mean $\operatorname{IgA}^{+}$ B ACC in TC was recorded in Experiment I nor in Experiment II.

In Experiment III a highly significant increase in $\mathrm{IgA}^{+} \mathrm{B}$ ACC was noted on $14 \mathrm{DPV}$ in both HG and TC in the MDA-0/V group (Table 1).

In comparison with experiment III, in Experiment IV no statistical differences in mean $\operatorname{IgA}^{+} \mathrm{B}$ ACC was noted in $\mathrm{HG}$ between the vaccinated and control group of birds. In this Experiment significant and highly significant increase in $\operatorname{IgA}^{+} \mathrm{B}$ ACC was recorded in the $\mathrm{MDA}^{+} 14 / \mathrm{V}$ group on days 7 and $14 \mathrm{PV}$ in $\mathrm{TC}$, respectively, in comparison with virus-free birds (Table 1).

\section{Contribution of $\mathrm{B} \mathrm{IgA}^{+}$specific cells}

The contribution of anti-aMPV $\mathrm{B} \mathrm{IgA}^{+}$specific cells in HG and TC of birds of Experiment III and I are summarized in Fig. 1.

As demonstrated in Fig. $1 \mathrm{~A}$ in Experiment III no statistical difference was recorded in mean percentage contribution of anti-aMPV $\mathrm{B} \mathrm{IgA}^{+}$specific cells in HG between the vaccinated and control group, but an increasing tendency (with peak $7 \mathrm{DPV}$ ) of this parameter was observed in the TRT vaccinated group. In the MDA-0/V group a highly significant increase in mean percentage contribution of anti-aMPV B $\operatorname{IgA}^{+}$ specific cells in TC was observed $14 \mathrm{DPV}$, in comparison with the control group (Figs. 1B, 3)

In comparison with Experiment III, in Experiment I no statistical differences or increasing tendency of mean percentage contribution of anti-aMPV $\mathrm{B} \mathrm{IgA}^{+}$ 
A

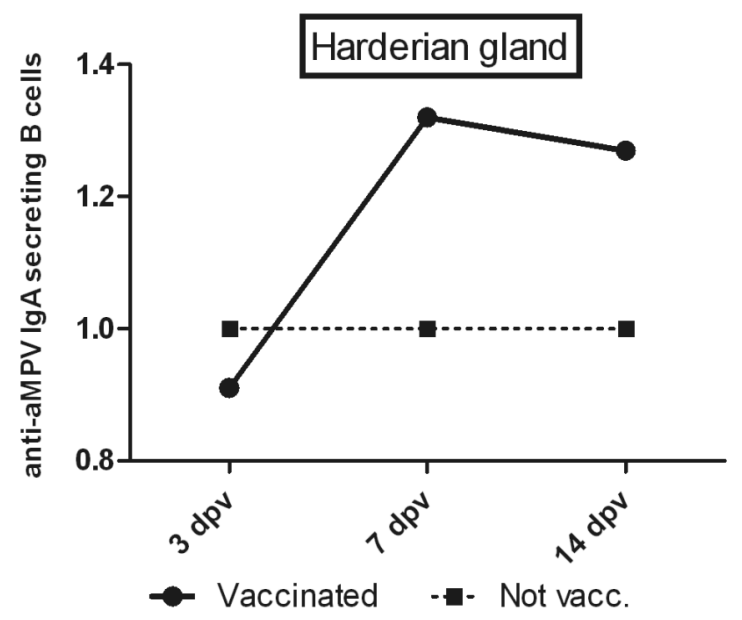

C

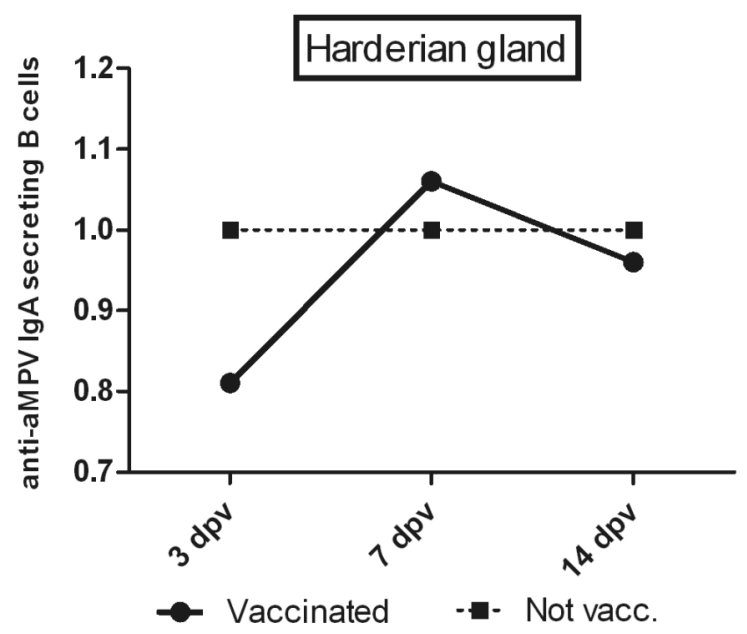

\section{B}

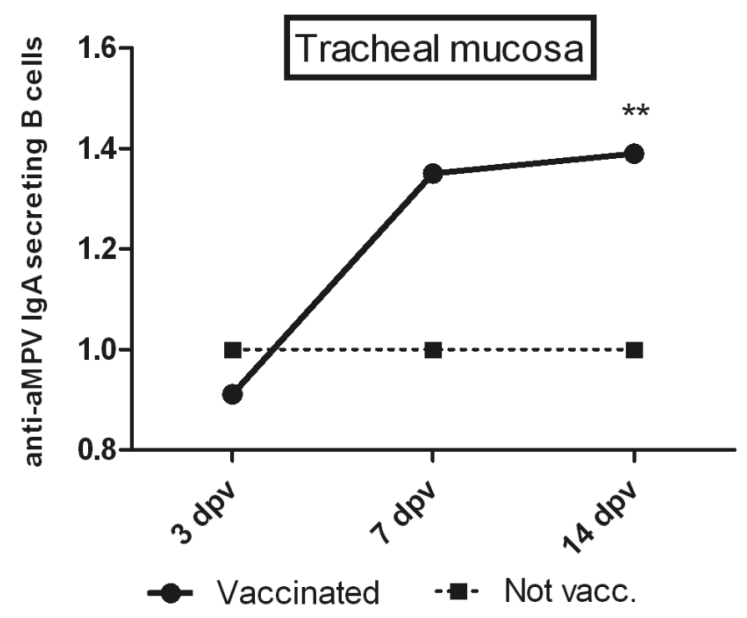

D

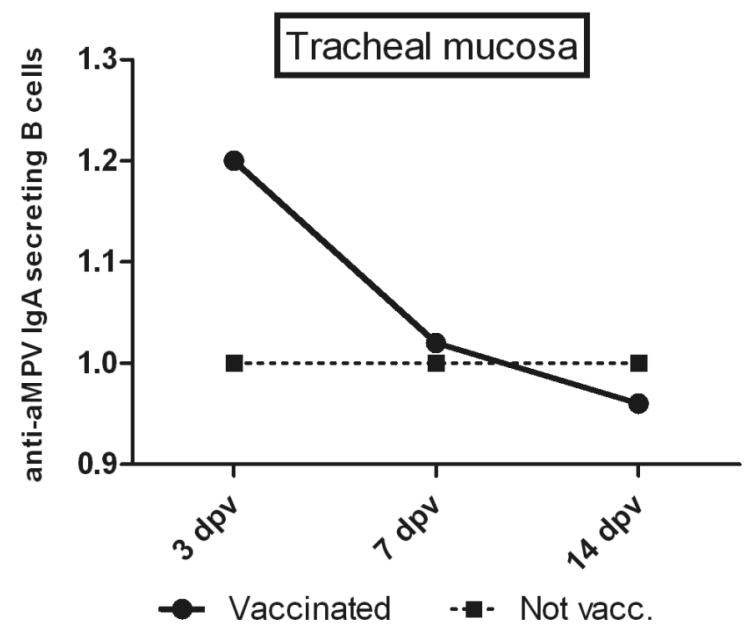

Fig. 1. Summary of contribution of anti-aMPV IgA ${ }^{+}$secreting B cells in $\mathrm{HG}$ and TC $(n=4)$ at different days post aMPV/A vaccination in Experiments I and III.

Results for vaccinated and not vaccinated (Not vacc.) groups in Experiment III (groups MDA-0/V and MDA-0/NV) for (A) HG and (B) TC; and Experiment I (groups $\mathrm{MDA}^{+} 0 / \mathrm{V}$ and $\mathrm{MDA}^{+} 0 / \mathrm{NV}$ ) for (C) $\mathrm{HG}$ and (D) TC.

Results for every experiment are presented as the mean $\mathrm{x}$-fold change of contribution of anti-aMPV IgA secreting $\mathrm{B}$ cells in vaccinated groups relative to control groups.

** Significant differences at different DPV $(T$-test, ** as $\mathrm{p}<0.01)$

specific cells in HG (Fig. 1 C) nor in TC (Fig. 1 D) was observed between the vaccinated and control group.

The contribution of anti-aMPV B IgA ${ }^{+}$specific cells in HG and TC of birds of Experiment IV and II are summarized in Fig. 2.

Despite the fact that in the vaccinated birds of Experiment IV a statistically significant decrease of the percentage contribution of anti-aMPV $\mathrm{B} \mathrm{IgA}^{+}$ specific cells was observed in TC, $3 \mathrm{DPV}$, in this group, on further examination days an increasing tendency of this parameter in both HG (3-14 DPV; Fig. $2 \mathrm{~A}$ ) and TC (7-14 DPV; Fig. 2 B) was observed in comparison to the control group, but the observed differences were not statistically significant.

In Experiment II no statistical differences in mean percentage contribution of anti-aMPV $\mathrm{B} \mathrm{IgA}^{+}$specific cells were observed between the vaccinated and control group in HG (Fig. 2C) or in TC (Fig. 2D), but an increasing tendency of this parameter was noted in the $\mathrm{MDA}^{+} 14 / \mathrm{V}$ group in HG (with peak $14 \mathrm{DPV}$; Fig. $2 \mathrm{C}$ ) in comparison with virus-free birds. 
A

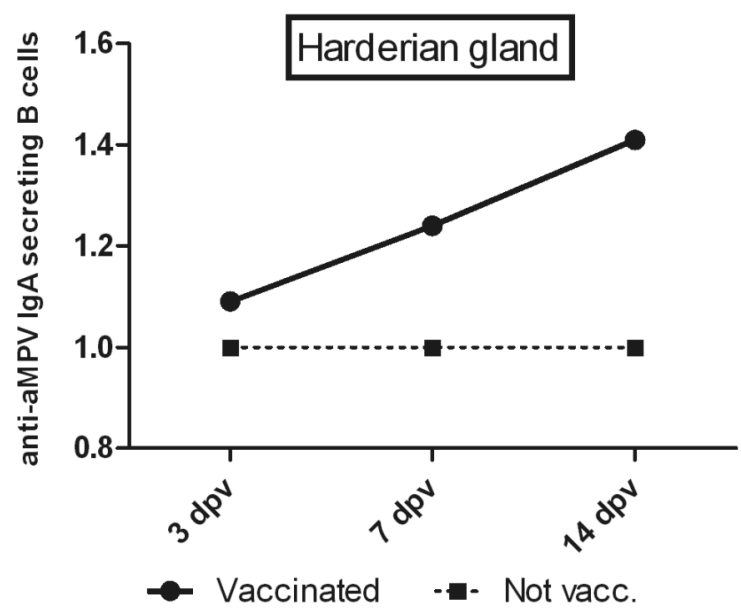

C

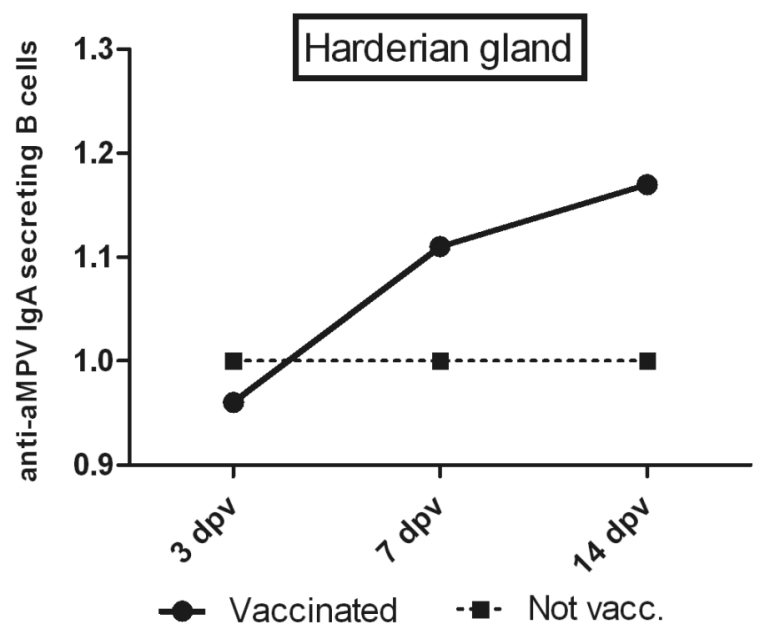

B

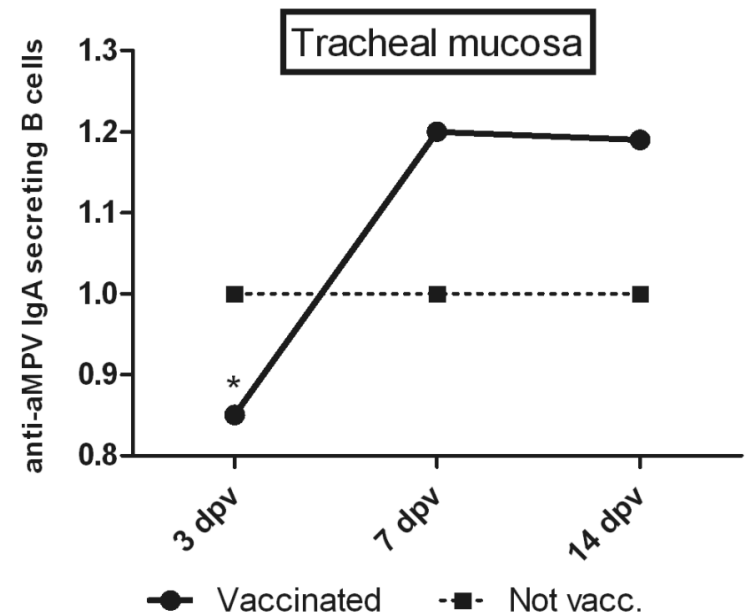

D

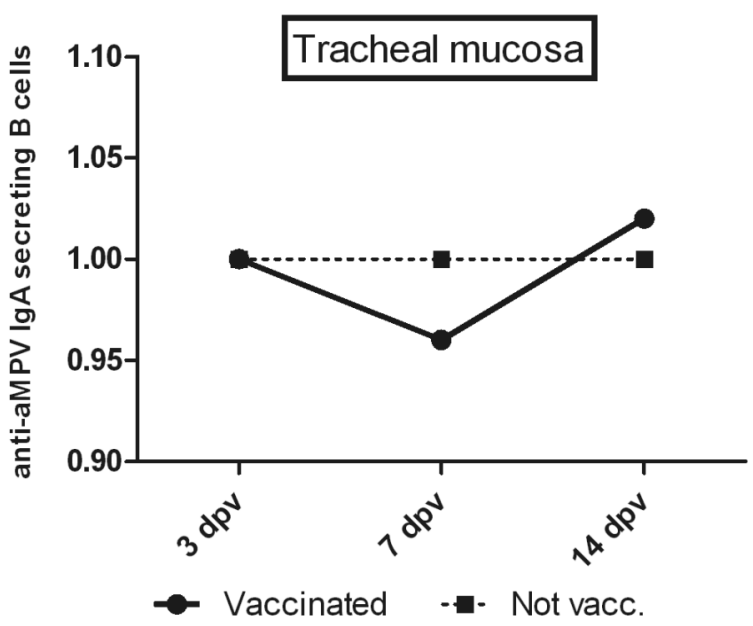

Fig. 2. Summary of contribution of anti-aMPV $\operatorname{IgA}^{+}$secreting $B$ cells in $H G$ and $T C(n=4)$ at different days post aMPV/A vaccination in Experiments II and IV.

Results for vaccinated and not vaccinated (Not vacc.) groups in Experiment IV (groups MDA-14/V and MDA-14/NV) for (A) HG and (B) TC; and Experiment II (groups MDA $14 / \mathrm{V}$ and $\mathrm{MDA}^{+} 14 / \mathrm{NV}$ ) for (C) HG and (D) TC.

Results for every experiment are presented as the mean $\mathrm{x}$-fold change of contribution of anti-aMPV IgA secreting B cells in vaccinated groups relative to control groups.

* Significant differences at different DPV $(T$-test, $*$ as $\mathrm{p}<0.05)$

\section{In-house ELISA}

No statistical differences in the level of anti - aMPV specific IgA antibodies in the serum were observed in any of the experiments (Table 2). Additionally, in Experiments I and II no statistical differences were observed in the level of IgA in tracheal washings (Table 3 ).

A gradual increase, with a statistically significant increase on days 7 (Experiment III) and $14 \mathrm{PV}$, in the level of anti-aMPV IgA in both Experiment III and
IV was observed in TRT vaccinated groups of turkeys (Table 3).

\section{Discussion}

TRT outbreaks are recorded frequently in the field, despite the fact that turkey poults are widely vaccinated against this disease on the first day of their life (Liman and Rautenschlein 2007, Catelli et al. 2010, Rautenschlein et al. 2011, Smialek et al. 2012). 
Table 2. Serum anti - aMPV IgA antibody level after aMPV vaccination ${ }^{\mathrm{a}}$ of turkeys.

\begin{tabular}{cccccc}
\hline \multirow{2}{*}{ Experiment } & \multirow{2}{*}{ Group } & \multicolumn{3}{c}{ Mean O.D. \pm S.D. ${ }^{\mathrm{b}}$ at days post aMPV/A vaccination } \\
\cline { 3 - 6 } & & 0 & 3 & 7 & 14 \\
\hline I & MDA+0/V (1:250) & $0.082 \pm 0.034$ & $0.068 \pm 0.032$ & $0.059 \pm 0.059$ & $0.05 \pm 0.008$ \\
& MDA+0/NV (1:250) & $0.082 \pm 0.034$ & $0.068 \pm 0.022$ & $0.053 \pm 0.006$ & $0.049 \pm 0.003$ \\
\hline III & MDA-0/V (1:250) & $0.048 \pm 0.004$ & $0.055 \pm 0.007$ & $0.049 \pm 0.004$ & $0.06 \pm 0.009$ \\
& MDA-0/NV (1:250) & $0.048 \pm 0.004$ & $0.054 \pm 0.006$ & $0.051 \pm 0.08$ & $0.06 \pm 0.011$ \\
\hline II & MDA+14/V (1:250) & $0.049 \pm 0.003$ & $0.051 \pm 0.005$ & $0.053 \pm 0.009$ & $0.047 \pm 0.003$ \\
& MDA+14/NV (1:250) & $0.049 \pm 0.003$ & $0.051 \pm 0.005$ & $0.051 \pm 0.008$ & $0.046 \pm 0.003$ \\
\hline IV & MDA-14/V (1:250) & $0.06 \pm 0.011$ & $0.057 \pm 0.008$ & $0.055 \pm 0.001$ & $0.051 \pm 0.005$ \\
& MDA-14/NV (1:250) & $0.06 \pm 0.011$ & $0.060 \pm 0.015$ & $0.055 \pm 0.008$ & $0.053 \pm 0.007$ \\
\hline
\end{tabular}

${ }^{a}$ In all experiments vaccinated birds were inoculated oculonasaly with $10^{4} \mathrm{TCID}_{50}$ of live attenuated aMPV/A vaccine

b 15 samples of serum were analyzed per group

${ }^{c}$ Sample dilution for ELISA procedure

Table 3. TW anti - aMPV IgA antibody level after aMPV vaccination ${ }^{\mathrm{a}}$ of turkeys.

\begin{tabular}{cccccc}
\hline \multirow{2}{*}{ Experiment } & \multirow{2}{*}{ Group } & \multicolumn{3}{c}{ Mean O.D. \pm S.D. ${ }^{\mathrm{b}}$ at days post aMPV/A vaccination } \\
\cline { 3 - 6 } & & 0 & 3 & 7 & 14 \\
\hline I & MDA+0/V & $0.084 \pm 0.029$ & $0.068 \pm 0.019$ & $0.063 \pm 0.01$ & $0.065 \pm 0.012$ \\
& MDA+0/NV & $0.084 \pm 0.029$ & $0.064 \pm 0.01$ & $0.068 \pm 0.016$ & $0.059 \pm 0.004$ \\
\hline III & MDA-0/V & $0.057 \pm 0.011$ & $0.078 \pm 0.006$ & $0.105 \pm 0.019^{*}$ & $0.11 \pm 0.026^{*}$ \\
& MDA-0/NV & $0.057 \pm 0.011$ & $0.076 \pm 0.012$ & $0.083 \pm 0.014$ & $0.091 \pm 0.009$ \\
\hline II & MDA+14/V & $0.059 \pm 0.004$ & $0.075 \pm 0.017$ & $0.061 \pm 0.007$ & $0.069 \pm 0.008$ \\
& MDA+14/NV & $0.059 \pm 0.004$ & $0.076 \pm 0.012$ & $0.067 \pm 0.012$ & $0.062 \pm 0.008$ \\
\hline IV & MDA-14/V & $0.091 \pm 0.009$ & $0.118 \pm 0.05$ & $0.09 \pm 0.026$ & $0.096 \pm 0.012^{*}$ \\
& MDA-14/NV & $0.091 \pm 0.009$ & $0.088 \pm 0.022$ & $0.073 \pm 0.017$ & $0.08 \pm 0.013$ \\
\hline
\end{tabular}

${ }^{a}$ In all experiments vaccinated birds were inoculated oculonasaly with $10^{4} \mathrm{TCID}_{50}$ of live attenuated aMPV/A vaccine

b 10 samples of undiluted tracheal washings per group were analyzed

* Significant difference in mean O.D. of vaccinated birds in comparison to the not vaccinated group (T-test, * as $\mathrm{p}<0.05)$.

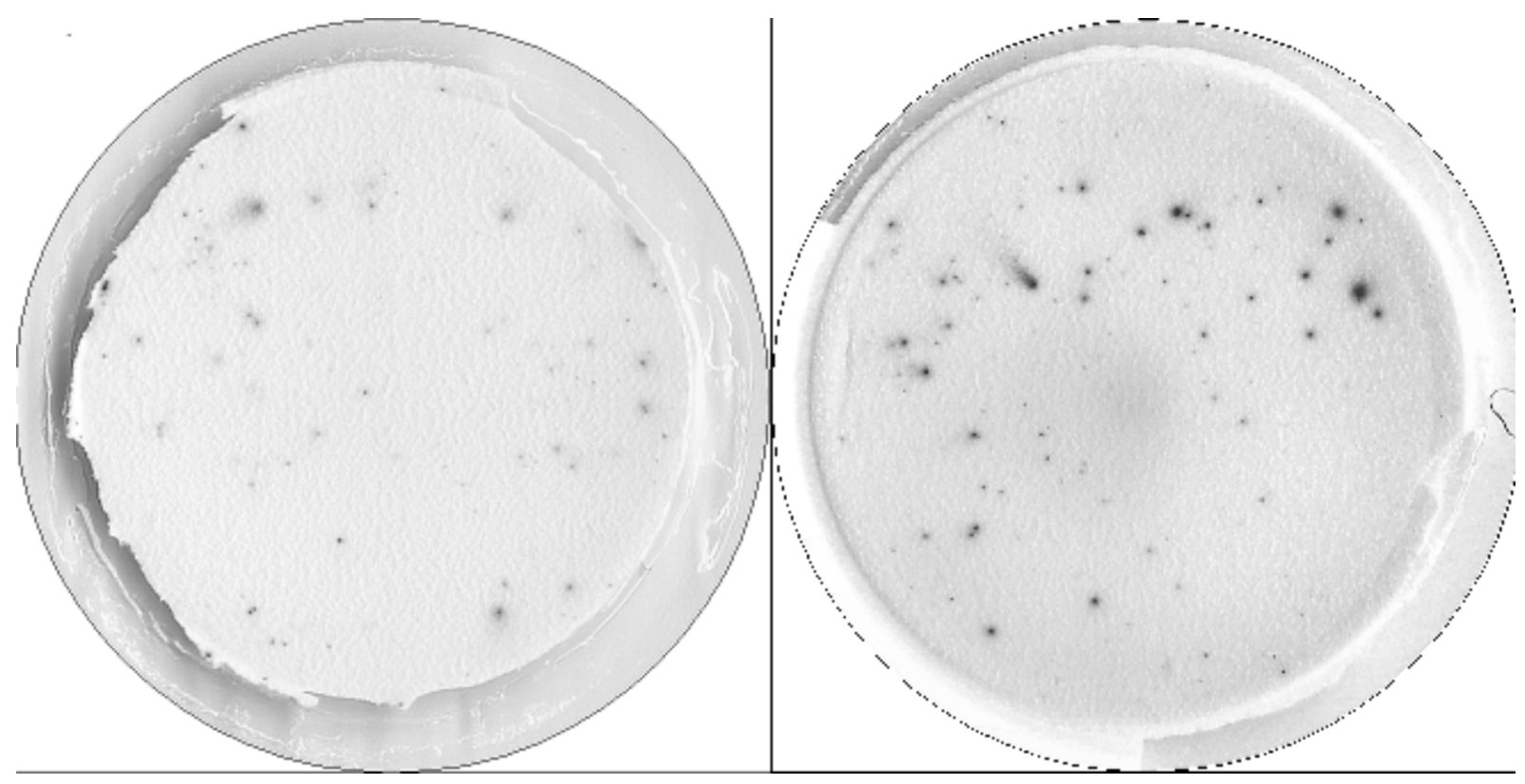

Fig. 3. On the left - anti-aMPV B IgA ${ }^{+}$SFU within lymphocytes isolated from TC of vaccinated birds in Experiment III, 14 DPV. On the right - total $\mathrm{B} \mathrm{IgA}^{+}$SFU within lymphocytes isolated from TC of vaccinated birds in Experiment III, 14 DPV. Contribution of anti-aMPV B $\operatorname{IgA}^{+}$memory cells was calculated with the use of the following formula: contribution of anti-aMPV IgA secreting B cells = (mean anti-aMPV B IgA ${ }^{+}$SFU / mean total B IgA $\left.{ }^{+} \mathrm{SFU}\right) * 100 \%$. Data were expressed as the mean $x$-fold change of contribution of anti-aMPV IgA secreting $\mathrm{B}$ in vaccinated groups relative to control groups. 
Cell-mediated immunity is increasingly often considered as the decisive factor in protection against TRT (Jones et al. 1992, Liman and Rautenschlein 2007, Smialek et al. 2012). In our previous study we demonstrated that stimulation of URT cell-mediated and humoral immunity (associated with IgY) after TRT vaccination depends on the level of anti - aMPV MDA on the day of TRT vaccination (Smialek et al. 2015).

Humoral immunity of bird URT is associated mainly with $\operatorname{sIg} \mathrm{A}$, and its protective role has been demonstrated based on several poultry respiratory infections (Jayawardane and Spradbrow 1995, Ganapathy et al. 2005, Van Ginkel et al. 2008). Up to date, little is known about the role of $\operatorname{sIgA}$ in the protection against TRT. Rautenschlein et al. (2011) reported that aMPV/A or B infection of chickens induces local production of IgA. Additionally, Cha et al. (2007) demonstrated a significant increase in the numbers of infiltrating $\operatorname{IgA}^{+} \mathrm{B}$ cells in nasal turbinates 7 days after the first aMPV/C exposure, with an elevated level of aMPV-specific IgA detected in the nasal secretions of virus-exposed birds after the second, but not after the first, virus exposure. These results indicate that $\operatorname{sIg} \mathrm{A}$ is involved in protection against TRT by suppressing aMPV replication in the URT and therefore it alleviates TRT course and enables faster recovery.

Mucosal production of IgA is T cell dependent (in vitro depletion of $\mathrm{TCR} \alpha \beta$ cells population decreases IgA production) (Cihak et al. 1991). Considering our previous findings concerning the differences in vaccine-induced humoral and cell-mediated immune parameter stimulation after TRT vaccination of turkeys of different immunological status (Smialek et al. 2015), the aim of this study was to investigate the impact of MDA on the stimulation, and antigen specificity acquisition of $\mathrm{B} \mathrm{IgA}^{+}$cells in the URT and the production of specific sIgA. In this study, $\operatorname{IgA}^{+} \mathrm{B}$ cells isolated from the $\mathrm{HG}$ and $\mathrm{TC}$ were subjected to three step quantitative-qualitative profile evaluation of: (I) production, (II) antigen specificity acquisition and (III) secretion of specific anti-aMPV IgA after TRT vaccination.

The ELISPOT technique that was developed in this study calculated the proportion of aMPV/A specific IgA-producing B cells within the total population of cells producing IgA. This calculation results from the fact that direct calculation of the absolute number of aMPV/A specific IgA-producing B cells is not a perfect indicator of immune memory acquisition, since the contribution of $\mathrm{IgA}^{+} \mathrm{B}$ cells may vary between the samples. Additionally, we have observed that B cells isolated from HG or TC samples of non vaccinated birds are also capable of producing $\operatorname{IgA}$ (specific or total $\operatorname{IgA}$ ) in vitro. Given the fact that the number of lymphocytes sorted into different ELISPOT plate wells was equal for every sample, the direct calculation could have given false positive results (in comparison to the control groups) for samples with a high percentage contribution of $\operatorname{IgA}^{+}$cells, and false negative results for samples with a low percentage contribution of $\operatorname{IgA}^{+} \mathrm{B}$ cells. In this study we evaluated the contribution of aMPV/A specific $\operatorname{IgA}^{+} \mathrm{B}$ cells in regard to the total number of $\operatorname{IgA}^{+} \mathrm{B}$ cells.

As it appears from our study, a high level of specific anti-aMPV IgY antibodies on the day of TRT vaccination of turkeys may have an impact on different levels of maturation of B lymphocytes into cells that produce and secrete IgA. Despite the fact that between 3-14 DPV in the $\mathrm{MDA}^{+} 0 / \mathrm{V}$ group an increase of $\mathrm{B} \mathrm{IgA}^{+} \mathrm{ACC}$ was recorded, no statistical differences (or increasing tendency) in the contribution of $\mathrm{B} \mathrm{IgA}^{+}$specific cells nor an increased level of specific IgA was observed in this group after vaccination. In the $\mathrm{MDA}^{+} 14 / \mathrm{V}$ group, a statistically significant increase of $\mathrm{B} \mathrm{IgA}^{+} \mathrm{ACC}(7 \mathrm{DPV})$ and an increasing tendency of the contribution of $\mathrm{B} \mathrm{IgA}^{+}$specific cells in HG was recorded, but we still did not observe the production of specific IgA after TRT vaccination. In Experiments I and II, an increase of $\mathrm{B} \operatorname{IgA}^{+} \mathrm{ACC}$ was demonstrated in HG (and not TC) after aMPV/A vaccination.

In comparison to $\mathrm{MDA}^{+}$vaccinated groups, in MDA- groups, regardless of the age on the day of TRT vaccination, an increase of mean $\mathrm{B} \mathrm{IgA}^{+} \mathrm{ACC}$ was delayed (between 7-14 DPV) and was observed mainly in TC. This character of B cell stimulation may have been associated with delayed infiltration of $\mathrm{T} \mathrm{CD}^{+}$cells (in MDA- groups) in the URT, which has been demonstrated previously (Smialek et al. 2015). Despite the above findings, in MDA- groups, a most prominent increasing tendency and/or statistically significant increase (in TC of MDA-0/V birds, 14 DPV) of the contribution of $\mathrm{B} \mathrm{IgA}^{+}$specific cells in $\mathrm{TC}$ and $\mathrm{HG}$ was observed after vaccination. Additionally, in Experiments III and IV, an increase in the level of specific IgA in tracheal washings (but not in serum) was demonstrated in vaccinated birds 7 DPV (Experiment III) and 14 DPV (Experiments III and IV).

If we combine flow cytometry, ELISPOT and ELISA results it is noted that $\mathrm{MDA}^{+}$vaccinated birds reacted with random multiplication (or infiltration) of $\operatorname{IgA}^{+} \mathrm{B}$ cells in URT, but the quality of such cells and their potential to produce anti-aMPV IgA remained unaltered. On the other hand, it took a longer time for MDA- vaccinated birds to mobilize $\operatorname{IgA}^{+} \mathrm{B}$ cells, but the population of these cells was characterized with high anti-aMPV IgA production capacity. 
The above data indicates that regardless of bird age on the day of TRT vaccination or aMPV subtype used, $\operatorname{Ig} \mathrm{A}^{+} \mathrm{B}$ cells and $\operatorname{IgA}$ are involved in protection against aMPV infection or re-infection. However, the stimulation of these parameters is influenced by the level of specific anti-aMPV IgY on the day of vaccination and, therefore, IgA secretion is more likely to be stimulated in MDA- birds after single TRT vaccination of turkey poults.

The mechanism of this adverse situation could be explained based on research into fragment crystallizable receptors $(\mathrm{FcR})$, characterized in humans. It has been reported that FcR may suppress the expression of humoral immunity (Ravetech and Lanier 2000) when a complex of IgG - antigen inhibits the activation of B lymphocytes through interaction with Fc $\gamma$ RII (FcR for $\gamma$ chain of IgG). No studies of FcR have been conducted in birds so far.

Finally, the results of this study corroborate our previous findings that the development of vaccine-induced immunity depends on the level of MDA and the age of birds on the day of vaccination against TRT (Smialek et al. 2015). In the light of the results of these studies we may conclude that a high level of MDA on the day of aMPV vaccination not only inhibits the infiltration of T cells in the URT, and replication of vaccine aMPV (Smialek et al. 2015) but it also causes a disturbance in antigenic specificity acquisition of B lymphocytes and anti-aMPV IgA production. These complex finding indicate that MDA could have an impact on TRT vaccination efficiency, which could in some part explain the observed frequency and the severity of TRT field outbreaks in vaccinated flocks.

Considering the fact that turkeys of Experiments I and II as well as III and IV had a different origin we were not able to perform statistical analysis of the analyzed parameters between them. To ensure research reproducibility PCR technique was implemented to ensure that experimental birds were free from respiratory tract pathogens such as Mycoplasma spp., Ornithobacterium rhinotracheale, Bordetella avium and Newcastle disease virus (data not shown).

\section{Acknowledgements}

This project was supported with funds from the National Science Centre granted in terms of decision number DEC-2011/01/N/NZ6/05757.

\section{References}

Buys SB, Du Preez JH (1980) A preliminary report on the isolation of a virus causing sinusitis in turkeys in South
Africa and attempts to attenuate the virus. Turkeys 28: 36.

Catelli E, Lupini C, Cecchinato M, Ricchizzi E, Brown P, Naylor CJ (2010) Field avian metapneumovirus evolution avoiding vaccine induced immunity. Vaccine 28: 916-921.

Cha RM, Khatri M, Sharma JM (2007) B-cell infiltration in the respiratory mucosa of turkeys exposed to subtype $\mathrm{C}$ avian metapneumovirus. Avian Dis 51: 764-770.

Cihak J, Hoffmann-Fezer G, Ziegler-Heitbrock HW, Stein H, Kaspers B, Chen CH, Cooper MD, Losch U (1991) $\mathrm{T}$ cells expressing the $\mathrm{V}$ beta $1 \mathrm{~T}$-cell receptor are required for IgA production in the chicken. Proc Natl Acad Sci USA 88: 10951-10955.

Collins MS, Gough RE, Alexander DJ (1993) Antigenic differentiation of avian pneumovirus isolates using polyclonal antisera and mouse monoclonal antibodies. Avian Pathol 22: 469-479.

Cook JK, Holmes HC, Finney PM, Dolby CA, Ellis MM, Huggins MB (1989) A live attenuated turkey rhinotracheitis virus vaccine. 2 . The use of the attenuated strain as an experimental vaccine. Avian Pathol 18: 523-534.

Cook JK, Jones BV, Ellis MM, Jing L, Cavanagh D (1993) Antigenic differentiation of strains of Turkey rhinotracheitis virus using monoclonal antibodies. Avian Pathol 22: 257-273.

Ganapathy K, Cargill P, Montiel E, Jones RC (2005) Interaction between live avian pneumovirus and Newcastle disease virus vaccines in specific pathogen free chickens. Avian Pathol 34: 297-302.

Jayawardane GW, Spradbrow PB (1995) Mucosal immunity in chickens vaccinated with the V4 strain of Newcastle disease virus. Vet Microbiol 46: 69-77.

Jones RC, Naylor CJ, al-Afaleq A, Worthington KJ, Jones $R$ (1992) Effect of cyclophosphamide immunosuppression on the immunity of turkeys to viral rhinotracheitis. Res Vet Sci 53: 38-41.

Jones RC, Williams RA, Baxter-Jones C, Savage CE, Wilding GP (1988) Experimental infection of laying turkeys with rhinotracheitis virus: distribution of virus in the tissues and serological response. Avian Pathol 17:841-850.

Liman M, Rautenschlein S (2007) Induction of local and systemic immune reactions following infection of turkeys with avian Metapneumovirus (aMPV) subtypes A and B. Vet Immunol Immunopathol 115: 273-285.

Pedersen JC, Reynolds DL, Ali A (2000) The sensitivity and specificity of a reverse transcription-polymerase chain reaction assay for the avian pneumovirus (Colorado strain). Avian Dis 44: 681-685.

Ratcliffe MJH, Hartle S (2014) B cells, the Bursa of Fabricius and the generation of antibody repertoires. In: Schat KA, Kaspers B, Kaiser P (eds) Avian Immunology 2nd edition, Academic Press, San Diego, pp 65-89.

Rautenschlein S, Aung YH, Haase C (2011) Local and systemic immune responses following infection of broiler-type chickens with avian Metapneumovirus subtypes A and B. Vet Immunol Immunopathol 140: 10-22.

Ravetch JV, Lanier LL (2000) Immune inhibitory receptors. Science 290: 84-89.

Smialek M, Pestka D, Tykalowski B, Stenzel T, Koncicki A (2015) Development of vaccine-induced immunity against TRT in turkeys depends remarkably on the level of maternal antibodies and the age of birds on the day of vaccination. BMC Vet Res 11: 28. 
Smialek M, Tykalowski B, Stenzel T, Koncicki A (2012) The perspective of immunoprophylaxis and selected immunological issues in the course of the turkey rhinotracheitis. Pol J Vet Sci 15: 175-180.

Smialek M, Tykalowski B, Stenzel T, Koncicki A (2011) Local immunity of the respiratory mucosal system in chickens and turkeys. Pol J Vet Sci 14: 291-297.
Snoeck V, Peters IR, Cox E (2006) The IgA system: a comparison of structure and function in different species. Vet Res 37: 455-467.

Van Ginkel FW, van Santen VL, Gulley SL, Toro H (2008) Infectious bronchitis virus in the chicken Harderian gland and lachrymal fluid: viral load, infectivity, immune cell responses, and effects of viral immunodeficiency. Avian Dis 52: 608-617. 